vaccination is the most effective method of preventing infectious diseases. The majority of parents (80\%) understand that vaccinated children are less likely to develop severe illness than those who have not been vaccinated. More than half of respondents (60\%) are not satisfied the quantity with quality of information about vaccinations obtained from the doctor.

Conclusion The findings suggest that the majority of parents have a generally positive attitude toward vaccination and consider it necessary. However, respondents showed insufficient awareness of vaccination problems. Therefore, educational events should be planned and new methods of work should be developed to raise awareness about vaccination among parents.

\section{P616 THE APPROACHES FOR EARLY DETECTION IMMUNE PERTURBATION IN CHILDREN LIVING IN URBAN AREAS WITH ADVERSE ENVIRONMENTAL}

${ }^{1}$ Alfia Aminova* ${ }^{2}$ Aleftina Akatova, ${ }^{2}$ Olga Vozgament, ${ }^{1}$ Zarnigar Gumbatova, ${ }^{1}$ Tatiana Eryushova, 'Galina Borodina. ${ }^{1}$ Federal State Autonomous Educational Institution of Higher Education I.M. Sechenov First Moscow State Medical University of the Ministry of Health of the Russian Federation (Sechenov University), Moscow, Russia, Moscow, Russian Federation; ${ }^{2}$ Federal Scientific Center for Medical and Preventive Health Risk Management Technologies, Perm, Russia, Perm, Russian Federation

10.1136/archdischild-2019-epa.947

Background The objective was to determine the approach of early detection immune perturbation in the children living under exposure to organic compounds with immunotoxicity. Although the impact of man-made environmental factors on the immune system is proven by clinical and immunological findings, many issues remain unsolved. Comprehensive studies analyzing humoral and cellular immunity are extensive, and few studies addressed definition of screening examination method.

Material and methods The comprehensive profound examination of 429 children at the age of 13-15 years living near the oil-refining complex has been carried out. All children were divided into groups based on the degree of man-made environmental factors impact and the ratio of echostructure changes in the spleen. The standard complex of clinical laboratory diagnostic technique was used in the presented study.

Results All the examined children have been divided into two groups.1st group comprised 256 children who had significant ultrasonic changes of the spleen (multiple hypoechogenic homogeneous inclusions throughout the ultrasonic section of the organ). In the 2 nd group (236 children) the ultrasonic examination had no disturbances of spleen (echostructure was homogeneous and fine-gritted). The chemical and analytical analys of children's blood of children have been obtained statistically significant differences of the number of indicators for immune profile of children without disorders of spleen's echostructure (2nd group) vs the 1 st group ( $\mathrm{p} \leq 0.05)$. It was determinated that children living in areas with technogenic pollution had significantly increased expression of CD19 +receptor and CD25 +-receptor on T-lymphocytes (by relative and absolute measure) compared to the children's group living within the territory of minimal technogenic pollution $(p<0.05)$. The cause-effect relations between the existing deviations of the immunograms and the content of ecotoxicants in the blood were proved by the mathematical model method.
Conclusion The diagnostic value of spleen's ultrasound spleen examination with the determination of the changes type in the parenchymatous tissue was determined and can be recommended as a screening method for assessment the impact of man-made environmental factors on the immune system of children living in urban areas.

\section{P617 PRIMARY IMMUNODEFICIENCIES (PID) OF THE CHILD: ABOUT 16 CASES}

${ }^{1}$ Bayene Maalej, 'Imene Moalla, 'Manel Wali, 'Muhamed Muzaker, ${ }^{2}$ Chiraz Regaieg*, ${ }^{2}$ Abdelatif Gargouri, ${ }^{1}$ Faiza Safi, ${ }^{1}$ Manel Hsairi, 'Lamia Gargouri, ${ }^{1}$ Abdelmaijid Mahfoudh. ${ }^{1}$ Pediatric Emergency and Resuscitation Departmt Hedi Chaker University Hospital, Sfax, Tunisia; ${ }^{2}$ Neonatology Departmt Hedi Chaker University Hospital, Sfax, Tunisia

\subsection{6/archdischild-2019-epa.948}

Introduction Primary immunodeficiency disorders (PIDs) in children are genetic diseases that affect adaptive or innate immunity. The importance and nature of the clinical signs depend on the deficit function predominantly within the immune system.

Objective To study the epidemiological, clinical, paraclinical, therapeutic and evolutionary characteristics of PID in children. Patients and methods A retrospective study of 16 cases of primary immunodeficiencies (PID) of the child collected at the pediatric emergency and resuscitation departmt Hedi Chaker University Hospital Sfax over a period of 15 years (20042019).

Results 14 boys and 2 girls, mean age 3 years (range 2 months to 10 years). A notion of consanguinity was present in 15 cases. The respiratory manifestations were revealing in 9 cases. Five patients had impaired expression of HLA class II molecules, 4 patients had agammaglobulinemia, 5 patients had chronic septic granulomatous disease, 2 patients had hyper IgM syndrome and one patient had ataxia telangiectasia. Therapeutically all our patients have benefited from symptomatic treatment based on veinoglobulins. The outcome was favorable in 6 patients, one patient had hematopoietic stem cell transplantation, 4 patients had frequent hospitalisations for infections, and 5 patients had died.

Conclusion A better knowledge and characterization of the different immunodeficiencies allows an improvement of the care of these patients both preventive and curative, adapted to the deficit, as well as a more precise genetic advice.

\section{P618 SYSTEMIC LUPUS ERYTHEMATOSUS OF THE CHILD: STUDY OF 6 CASES}

${ }^{1}$ Bayene Maalej, ${ }^{1}$ Imene Moalla, ${ }^{1}$ Manel Wali, ${ }^{1}$ Sahar Trichilli, ${ }^{2}$ Manel Charfi', ${ }^{2}$ Abdeltif Gargouri, ${ }^{1}$ Faiza Safi, ${ }^{1}$ Manel Hsairi, ${ }^{1}$ Lamia Gargouri, 'Abdelmajid Mahfoudh. ${ }^{1}$ Pediatric Emergency and Resuscitation Departmt Hedi Chaker University Hospital, Sfax, Tunisia; ${ }^{2}$ Neonatology Departmt Hedi Chaker University Hospital, Sfax, Tunisia

\subsection{6/archdischild-2019-epa.949}

Objective To study the clinical, therapeutic and progressive features of systemic lupus erythematosus (SLE) in children.

Patients et methods A retrospective study of 5 cases of childhood SLEs collected at the Pediatric Emergency and Resuscitation Department Hedi Chaker University Hospital Sfax over a period of 13 years (2006-2018).

Results These are 3 girls and 3 boys. The age of onset of symptomatology ranged from 9 years to 13 years. The telltale 